\title{
Associations of dietary patterns with bone mass, muscle strength and balance in a cohort of Australian middle-aged women
}

\author{
Feitong Wu ${ }^{1}$, Karen Wills ${ }^{1}$, Laura L. Laslett ${ }^{1}$, Brian Oldenburg ${ }^{2}$, Graeme Jones ${ }^{1}$ and Tania Winzenberg ${ }^{1,3 *}$ \\ ${ }^{1}$ Menzies Institute for Medical Research, University of Tasmania, 17 Liverpool Street, Hobart, TAS 7000, Australia \\ ${ }^{2}$ School of Population and Global Health, University of Melbourne, 235 Bouverie Street, Carlton, VIC 3053, Australia \\ ${ }^{3}$ Faculty of Health, University of Tasmania, 17 Liverpool Street, Hobart, TAS 7000, Australia \\ (Submitted 12 December 2016 - Final revision received 26 July 2017 - Accepted 22 August 2017 - First published online 9 October 2017)
}

\section{Abstract}

Influences of dietary patterns on musculoskeletal health are poorly understood in middle-aged women. This cross-sectional analysis from a cohort of 347 women (aged 36-57 years) aimed to examine associations between dietary patterns and musculoskeletal health outcomes in middle-aged women. Diet was measured by the Cancer Council of Victoria FFQ. Total body bone mineral content (TB BMC), femoral neck and lumbar spine bone density (dual-energy X-ray absorptiometry), lower limbs muscle strength (LMS) and balance tests (timed up and go test, step test, functional reach test (FRT) and lateral reach test) were also measured. Exploratory factor analysis was used to identify dietary patterns and scores for each pattern generated using factor loadings with absolute values $\geq 0 \cdot 20$. Associations between food pattern scores and musculoskeletal outcomes were assessed using multivariable linear regression. Three dietary patterns were identified: 'Healthy' (high consumption of a plant-based diet - vegetables, legumes, fruit, tomatoes, nuts, snacks, garlic, whole grains and low intake of high-fat dairy products), 'high protein, high fat' (red meats, poultry, processed meats, potatoes, cruciferous and dark-yellow vegetables, fish, chips, spirits and high-fat dairy products) and 'Processed foods' (high intakes of meat pies, hamburgers, beer, sweets, fruit juice, processed meats, snacks, spirits, pizza and low intake of cruciferous vegetables). After adjustment for confounders, Healthy pattern was positively associated with LMS, whereas Processed foods pattern was inversely associated with TB BMC and FRT. The associations were not significant after accounting for multiple comparisons. There were no associations with any other outcomes. These results suggest that maintaining a healthy diet could contribute to bone acquisition, muscle strength and balance in adult life. However, while they provide some support for further investigating dietary strategies for prevention of age-related loss of muscle and deterioration in balance, the exploratory nature of the analyses means that confirmation in longitudinal studies and/or trials with pre-specified hypotheses is needed.

\section{Key words: Dietary patterns: Bone mass: Muscle strength: Balance: Middle-aged women}

Osteoporosis is a major public health issue worldwide. It results in an increased susceptibility to fractures, which in turn lead to lower quality of life ${ }^{(1)}$, and increased disability and mortality ${ }^{(2)}$. Of note, low BMD is a major risk factor for fracture throughout the lifetime ${ }^{(4,5)}$. BMD in adults depends on both peak bone mass that is achieved at around the third decade of life and the rate of bone loss subsequently ${ }^{(6,7)}$. Therefore, creating a greater reserve of bone mass at a younger age and slowing bone loss throughout adult life are critical parts of any strategy for preventing future osteoporotic fractures.

Falls are also a major fracture risk factor - more than $90 \%$ of hip fractures are the result of a fall ${ }^{(3)}$ and falls cause a high rate of mortality ${ }^{(4)}$. Poor balance is a primary risk factor of falling in older adults ${ }^{(5)}$. In addition, muscle weakness is associated with reduced balance in the older population ${ }^{(6,7)}$ and progressive loss of muscle strength (especially of the lower limbs) with aging is also a major risk factor for falls ${ }^{(8,9)}$. Importantly, both muscle strength and balance decline dramatically between 45 and 55 years of age $\mathrm{e}^{(10,11)}$, suggesting there could be potential benefits of early interventions during this age period.

Studies focussing on particular nutrients (e.g. Ca and vitamin D), foods or food groups (e.g. dairy products) have identified associations between individual nutrients and musculoskeletal health outcomes ${ }^{(12,13)}$. In particular, studies have demonstrated beneficial associations of sufficient nutrients (e.g. protein, vitamin D and antioxidant nutrients) with BMD, muscle strength and balance ${ }^{(13-15)}$. However, such approaches of using individual nutrients or food items do not consider interactions, intercorrelations and cumulative effects between different nutrients and foods ${ }^{(16)}$. Dietary pattern analysis has been used as an alternative to deal with these limitations by studying the overall diet rather than intakes of specific individual nutrients.

Abbreviations: FRT, functional reach test; LMS, limb muscle strength; TB BMC, total body bone mineral content.

* Corresponding author: T. Winzenberg, fax +61 36226 7704, email Tania.Winzenberg@utas.edu.au 
This is particularly important for disease prevention or treatment because the effect of a single or a few nutrients may be too small to be detectable while dietary pattern analysis considers the joint effects of nutrients and foods based on entire eating pattern/behaviour. The results of dietary patterns studies can also be easy for the public to translate into diets and refine the dietary guidelines in the prevention of diseases.

The links between dietary patterns and bone health have largely been investigated in the elderly ${ }^{(17-19)}$, but the results might not be generalisable to younger adults. Indeed, conflicting conclusions have been drawn in the literature on the relationship between a 'Western' dietary pattern and bone health among younger ${ }^{(17,20)}$ and older adults ${ }^{(17-19)}$, which might be due to the change in diet behaviours and quantity of foods/ nutrients for improving musculoskeletal health. In contrast, only a few studies have examined the association of dietary patterns and musculoskeletal health outcomes in younger adults ${ }^{(17,20,21)}$, showing an inconclusive association of various dietary patterns with bone health. However, these studies in younger adults have assessed bone density outcomes ${ }^{(17,20,21)}$ but not muscle strength or balance. Therefore, this study aimed to assess the association of dietary patterns identified by factor analysis with multiple musculoskeletal outcomes, including bone density, lower limb muscle strength and balance in a cohort of Australian women aged 36-57 years.

\section{Methods}

\section{Study population}

The sample for this cross-sectional study comprised 347 women (aged 36-57 years) who participated in a 10-year additional follow-up of a 2-year osteoporosis randomised controlled trial in Southern Tasmania, Australia, with details reported previously $^{(22)}$. In brief, women aged 25-44 years were randomly selected from the Tasmanian Electoral Roll in 2000. Women were recruited if they were free of the following: previous measurement of BMD (as the intervention involved used bone density to provide feedback to participants of their relative risk of fractures in later life), history of thyroid disease, renal failure, malignancy, rheumatoid arthritis, hysterectomy, hormone replacement therapies, pregnancy or planning pregnancy within 2 years of study entry, or lactating. At baseline, 470 women were randomly assigned to one of two osteoporosis educational interventions: group education using the Osteoporosis Prevention and Self-management course (OPSMC) or an information leaflet. The OPSMC is a chronic disease selfmanagement course developed by the Arthritis Foundation of Victoria and utilised by Osteoporosis Australia. It aims to increase knowledge, improve confidence and awareness and self-management of osteoporosis prevention with an emphasis on promoting appropriate lifestyle changes. The osteoporosis information leaflet, from Osteoporosis Australia 'Understanding Osteoporosis', provided a comprehensive description of osteoporosis and a discussion of the role of lifestyle factors including diet, exercise and smoking, and optimal levels of $\mathrm{Ca}$ intake and exercise. Participants had their BMD measured at the spine and hip at baseline, 2 and 12 years. At baseline, those with a mean spine and hip $T$ score $<0$ were informed that they were at a higher risk in later life whereas those with a mean $T$ score of 0 or greater were informed that they were not at higher risk (here termed as fracture risk feedback). At 12 years, all women from the original study were contacted to ask for participation in this cross-sectional study. For both the original randomised controlled trial and the present cross-sectional study, ethics approval was obtained from the Tasmania Health and Medical Human Research Ethics Committee (EC00337) and all participants gave written informed consent. A total of 347 women who attended the 12-year follow-up and had at least one of the outcomes were included in this cross-sectional analysis, and the methods for the measures are described below.

\section{Bone mass}

Total body bone mineral content (TB BMC) and BMD at the lumbar spine (LS) and femoral neck (FN) were measured by dual-energy X-ray absorptiometry using fan beam setting on an in-house Hologic Delphi bone densitometer (Hologic QDR2000; Hologic), calibrated daily with CV $1 \%$.

\section{Balance}

We measured four clinical balance tests: the timed up and go test (TUG), the step test (ST), the functional reach test (FRT) and the lateral reach test (LRT). These tests assess balance performance from either a static or dynamic aspect, and are able to differentiate between 'fallers' and 'non-fallers' in older adults ${ }^{(23)}$. All have been validated in older women and have a high reliability, with normative values determined in women of the age in our study ${ }^{(23-25)}$.

$\mathrm{TUG}^{(26)}$ is a test of dynamic steady-state balance and gait. Participants sat in an armchair ( $45 \mathrm{~cm}$ high) with their back against the chair, then stood without using the arms, walked three metres, turned, walked back, and sat down. The average time of two trials was used for analysis.

The $\mathrm{ST}^{(27)}$ measures speed of performing a dynamic stepping task. Participants stood $5 \mathrm{~cm}$ from an $8.5-\mathrm{cm}$-high block positioned against a wall and placed the whole foot of one leg onto the block and then returned it to the floor repeatedly as fast as possible for $15 \mathrm{~s}$. The number of steps was recorded. Both sides were tested, and the mean number of steps for each side was calculated for analysis.

The FRT measures ability to reach forward with each arm from a bilateral stance position ${ }^{(24)}$. Participants stood with feet a comfortable distance apart behind a line perpendicular and adjacent to a wall. The arm closest to the wall was raised to shoulder height and the position of the knuckle of the middle finger marked ${ }^{(24)}$. Participants leaned forward as far as possible and distance of the knuckle from the first mark is recorded. The mean of three trials on each side was calculated for analysis.

The LRT measures ability to reach to the side in a bilateral stance $^{(25)}$. Participants stood with their backs near but not touching a wall with the heels $10 \mathrm{~cm}$ apart. Participants raised both arms to shoulder height while the tip of the third finger on the side being measured was marked. Participants then lowered the arm 
not being measured and reached sideways as far as possible with the arm being measured. The position of furthest reach was marked and the difference between the two marks calculated. The mean of three trials on each side was calculated for analysis.

\section{Lower limb muscle strength}

Lower limb muscle strength (LMS) was measured to the nearest kilogram using a dynamometer (TTM Muscular Meter) ${ }^{(28)}$ to assess isometric strength, predominantly of the quadriceps and hip extensors. The examiner demonstrated the correct technique to the participant before testing. Participants stood on the back of the dynamometer platform, with back against a wall and knees flexed to an angle of $115^{\circ}$. They held a bar, connected to the dynamometer by a chain, and lifted the bar using maximum force using their legs, with the back and neck straight. Two readings were made, and the mean calculated for analysis. The intra-class correlation coefficient for LMS was 0.94 $(95 \%$ CI $0.92,0.95)$ in this study (from two-way random-effects model $\left.^{(29)}\right)$.

\section{Dietary food intakes}

Dietary food intakes were measured using the AntiCancer Council of Victoria FFQ (CCV FFQ) and participants were asked to answer questions about their usual eating habits over the past 12 months. The details of the CCV FFQ have been described elsewhere ${ }^{(30,31)}$. In brief, this FFQ includes questions on 101 food and drink items. Participants were asked about the consumption of foods in four main categories (cereal foods, sweets and snacks; dairy products, meat and fish; fruit; vegetables) with frequency options ranging from never to three or more times per day, and detailed information on alcohol consumption. Also assessed were the number of pieces of fresh fruit and types of vegetables consumed daily, the type and daily amount of milk and bread eaten, type of spread put on bread, amount of sugar consumed daily, eggs eaten per week and type of cheese eaten, and serving sizes of potato, vegetables, steak and meat or vegetable casserole. The nutrients content of food were determined by Australian food composition tables ${ }^{(32)}$.

\section{Other measurements}

Strenuous and light physical activity levels were measured by a validated questionnaire ${ }^{(33)}$, which was modified for Tasmanian conditions and has been used previously in women of this age, where physical activity was related to bone mass of the $\mathrm{FN}^{(34)}$. Participants were asked how many days in the last fortnight did they do at least $20 \mathrm{~min}$ of strenuous exercise, measured in five categories $(1=0 \mathrm{~d}, 2=1-2 \mathrm{~d}, 3=3-5 \mathrm{~d}, 4=6-8 \mathrm{~d}, 5=\geq 9 \mathrm{~d})$. Frequency of light physical activity was asked in the same way. Height was measured by a stadiometer (The Leicester height measure, Invicta Plastics Ltd) and weight by a single set of calibrated scales (Heine). BMI was calculated (weight/height $\left(\mathrm{kg} / \mathrm{m}^{2}\right)$ ). We used a standardised questionnaire to collect smoking history (current/former/never), breast-feeding history (yes/no), number of children, family history of osteoporosis and/or fracture, and previous fractures, education level, employment status of main financial provider in the household, menopausal status, and marital status.

\section{Statistical analysis}

We had a power of $0 \cdot 8$ to detect a correlation of $0 \cdot 15$ at a Type 1 error of $5 \%$. We are not aware of any published data on the size of correlations to be expected between dietary pattern and bone density, muscle strength and balance, but this effect size is small so we are unlikely to be failing to detect any meaningful effect. For example, it is much smaller than the correlation between vitamin $\mathrm{D}$ and bone density ( $r 0.354$ for lumber spine and 0.305 for $\mathrm{FN}$ in postmenopausal women) ${ }^{(35)}$ and muscle strength ( $r 0.37$ in older adults) ${ }^{(36)}$, and comparable with the correlation with balance $(r 0 \cdot 17$ for gait speed and $0 \cdot 14$ for the Romberg balance test in older women $)^{(37)}$. Participant characteristics were summarised using means and standard deviations, range (continuous variable) or frequency (\%). We classified 101 food and drink items into thirty-three pre-defined food groups as had previously been done in an Australian cohort using the same FFQ, in which a western dietary pattern was associated with greater cognitive decline (online Supplementary Table S1) ${ }^{(38)}$. The thirty-three groups were used to derive dietary patterns using exploratory factor analysis, which is commonly used to identify underlying structure (i.e. pattern) of the large number of food groups and is particularly useful when there is no a pre-defined idea about the dietary patterns as compared with other techniques. Factor loadings were extracted using the principal component method with varimax rotation to produce orthogonal factors. Factor loadings are interpreted as correlations between food items and derived factors. We determined the number of main dietary patterns based on eigenvalues $>1 \cdot 25$, identification of break-point in the Scree plot and interpretability. Three major dietary patterns were extracted. We used weighted sum scores to calculate dietary pattern scores for each participant, as estimates of the underlying factor values. Items with factor loadings with absolute factor loadings $\geq 0.20$ were considered as significantly contributing to a dietary pattern, and were included in the dietary pattern score calculations. Factor loading cut-points used in the literature are arbitrary, but generally range from $0 \cdot 2$ to $0 \cdot 3$. We used 0.2 as this could include more important food groups in defining the patterns, which would give us a broader picture of the patterns ${ }^{(39)}$. Foods that had factor loadings $<0 \cdot 20$ were excluded from the pattern score calculation.

The associations between dietary pattern scores and musculoskeletal outcomes were estimated using multivariable linear regression with adjustment for confounders. We considered adjustment for both education intervention group and for risk feedback group (i.e. of high or normal risk of fracture). The former was considered in our process for selecting confounders, described below. However, the latter was determined by baseline BMD and so was highly correlated with BMD. Therefore, we could not adjust BMD in models for fracture risk feedback group. Also, at baseline, unsurprisingly, being in the high $v$. normal risk feedback group, that is in the lower half of the BMD distribution was strongly associated with weight, height, BMI and lower limb strength. As we already adjusted 
for BMI in our models, further adjusting for risk feedback group would be an overadjustment. Furthermore, risk feedback group was not associated with change in lower limb muscle strength at 2 years. Thus we did not adjust for fracture risk feedback group.

We selected other potential confounders (including education intervention) based on the biological plausibility of an association of a factor with both the outcome and the exposure of interest. Thus we considered age, menopausal status, weight, height, educational intervention, total energy intake, Ca and vitamin D supplement use, education level, employment status, marriage status, strenuous physical activity, hours of watching television, current smoking status, current use of oestrogens and oral contraceptive pill, history and total years of taking oral contraceptive, history of fractures and family history of osteoporosis and fractures as potential confounders. Weight, height and total energy intake were included in all models. Other factors were retained in the final model for each outcome when the estimated coefficient of each dietary pattern for that outcome changed by more than $10 \%$. Standardised dietary pattern scores were used in regressions, so the $\beta$-coefficients are interpreted as the change in the predicted value of the outcome for a standard deviation increase in the dietary pattern scores. Partial correlation coefficients between each dietary pattern score and energy-adjusted nutrient intake were calculated with adjustment for age. A two-tailed $P<0.05$ was considered significant, except the partial correlation between dietary patterns scores and nutrient intake, where a correlation coefficient with an absolute value of $>0.2$ was considered significant as described in previous literature ${ }^{(20)}$. To account for multiple testing, a critical value of $0.006(0.05 / 8)$ for statistical significance was subsequently applied. All analyses were performed in Stata version 12 (Stata Corporation).

\section{Results}

Baseline characteristics of participants who did and did not complete the 12-year follow-up have been previously reported ${ }^{(40)}$. In brief, compared with those who remained in the study, women lost to follow-up (26\%) were younger, had lower levels of educational attainment, and were more likely to be current smokers or to have ever smoked, and less likely to be married or in a de facto relationship. Other anthropometric and demographic factors were comparable. Table 1 shows characteristics of the study participants including intakes of nutrients.

Three dietary patterns were identified (Table 2). We labelled dietary pattern 1 as 'Healthy' because it was characterised by a more plant-based diet, with high intakes of vegetables (darkyellow, green leafy, cruciferous, legumes, garlic and others), fruit, tomatoes, nuts, snacks, whole grains. We labelled dietary pattern 2 as 'high protein, high fat' because of its high consumption of red meats, poultry, processed meats, potatoes, cruciferous and dark-yellow vegetables, fish, chips, spirits and high-fat dairy products. We labelled dietary pattern 3 as 'Processed foods' because it was characterised by high intakes of meat pies, hamburgers, beer, sweets, fruit juice, processed meats, snacks, spirits, pizza and low intakes of cruciferous vegetables. Mean of pattern scores were 194 (SD 122), 147
Table 1. Characteristics of study participants (women, $n$ 347) (Mean values and standard deviations; numbers and percentages)

\begin{tabular}{|c|c|c|}
\hline Characteristics & Mean & SD \\
\hline Age (years) & 49.9 & $5 \cdot 2$ \\
\hline Height $(\mathrm{cm})$ & $164 \cdot 0$ & $6 \cdot 1$ \\
\hline Weight (kg) & 73.8 & $15 \cdot 8$ \\
\hline BMI $\left(\mathrm{kg} / \mathrm{m}^{2}\right)$ & $27 \cdot 5$ & $5 \cdot 8$ \\
\hline Strenuous activity level ( $d / 2$ weeks) & $3 \cdot 0$ & 1.4 \\
\hline Serum 25(OH)D level (nmol/l) & $63 \cdot 1$ & $22 \cdot 8$ \\
\hline \multicolumn{3}{|l|}{ Vitamin D supplement use } \\
\hline$n$ & \multicolumn{2}{|c|}{126} \\
\hline$\%$ & \multicolumn{2}{|c|}{36} \\
\hline Dietary Ca intake (mg/d) & 1184 & 494 \\
\hline \multicolumn{3}{|l|}{ Ca supplement use } \\
\hline$n$ & \multicolumn{2}{|c|}{123} \\
\hline$\%$ & \multicolumn{2}{|c|}{35} \\
\hline \multicolumn{3}{|l|}{ Menopausal status } \\
\hline \multicolumn{3}{|l|}{ Postmenopause } \\
\hline$n$ & \multicolumn{2}{|c|}{86} \\
\hline$\%$ & \multicolumn{2}{|c|}{25} \\
\hline \multicolumn{3}{|l|}{ Premenopause } \\
\hline$n$ & \multicolumn{2}{|c|}{133} \\
\hline$\%$ & \multicolumn{2}{|c|}{38} \\
\hline Status unclear & & \\
\hline$n$ & & \\
\hline$\%$ & & \\
\hline Perimenopause & & \\
\hline$n$ & & \\
\hline$\%$ & & \\
\hline Employment status & & \\
\hline Unemployed & & \\
\hline$n$ & & \\
\hline$\%$ & & \\
\hline$<20 \mathrm{~h} /$ week & & \\
\hline$n$ & & \\
\hline$\%$ & & \\
\hline$>20 \mathrm{~h} /$ week & & \\
\hline$n$ & & \\
\hline$\%$ & & \\
\hline Educational levels & & \\
\hline$<$ Grade 10 & & \\
\hline$n$ & & \\
\hline$\%$ & & \\
\hline Grade $10-12$ & & \\
\hline$n$ & & \\
\hline$\%$ & & \\
\hline University & & \\
\hline$n$ & & \\
\hline$\%$ & & \\
\hline Watching television $(\mathrm{h} / \mathrm{d})$ & 2.9 & 0.7 \\
\hline Timed up and go test (s) & $5 \cdot 31$ & 0.71 \\
\hline Step test (steps) & $18 \cdot 4$ & $2 \cdot 6$ \\
\hline Functional reach test $(\mathrm{cm})$ & $41 \cdot 2$ & $6 \cdot 3$ \\
\hline Lateral reach test $(\mathrm{cm})$ & $18 \cdot 7$ & 3.9 \\
\hline Lower limb muscle strength $(\mathrm{kg})$ & $75 \cdot 6$ & $25 \cdot 5$ \\
\hline TB BMC $(g)$ & 2288 & 324 \\
\hline Femoral neck BMD $\left(\mathrm{g} / \mathrm{cm}^{2}\right)$ & 0.814 & 0.125 \\
\hline Lumbar spine BMD $\left(\mathrm{g} / \mathrm{cm}^{2}\right)$ & 1.035 & 0.151 \\
\hline Energy $(\mathrm{kJ} / \mathrm{d})$ & 6491 & 2111 \\
\hline Nutrients & & \\
\hline Carbohydrate $(\mathrm{g} / \mathrm{d})$ & 165 & 56 \\
\hline Protein $(g / d)$ & 80 & 31 \\
\hline Fat $(g / d)$ & 64 & 25 \\
\hline Cholesterol (mg/d) & 268 & 127 \\
\hline $\mathrm{Fe}(\mathrm{mg} / \mathrm{d})$ & 11.4 & $4 \cdot 3$ \\
\hline $\mathrm{Mg}(\mathrm{mg} / \mathrm{d})$ & 272 & 94 \\
\hline$P(\mathrm{mg} / \mathrm{d})$ & 1438 & 491 \\
\hline $\mathrm{Na}(\mathrm{mg} / \mathrm{d})$ & 2012 & 772 \\
\hline Vitamin C (mg/d) & 99 & 43 \\
\hline Vitamin $E(\mathrm{mg} / \mathrm{d})$ & $5 \cdot 8$ & $2 \cdot 3$ \\
\hline $\mathrm{Zn}(\mathrm{mg} / \mathrm{d})$ & $10 \cdot 3$ & 3.6 \\
\hline
\end{tabular}

TB BMC, total body bone mineral content; BMD, bone mineral density. 
Table 2. Rotated factor loadings for the three dietary patterns identified from exploratory factor analysis ( $n$ 347)

\begin{tabular}{|c|c|c|c|}
\hline Food groups & $\begin{array}{l}\text { 'Healthy' } \\
\text { pattern }\end{array}$ & $\begin{array}{l}\text { 'High protein, high } \\
\text { fat' pattern }\end{array}$ & $\begin{array}{l}\text { 'Processed } \\
\text { foods' pattern }\end{array}$ \\
\hline Dark-yellow vegetables & 0.61 & 0.42 & -0.14 \\
\hline Other vegetables & 0.58 & 0.09 & -0.06 \\
\hline Legumes & 0.57 & -0.19 & 0.07 \\
\hline Fruit & 0.49 & -0.11 & $-0 \cdot 15$ \\
\hline Tomatoes & 0.49 & $-0 \cdot 10$ & $0 \cdot 16$ \\
\hline Green leafy vegetables & 0.42 & 0.07 & -0.09 \\
\hline Cruciferous vegetables & 0.38 & 0.42 & -0.21 \\
\hline Nuts & 0.39 & $-0 \cdot 10$ & $-0 \cdot 11$ \\
\hline Snacks & 0.34 & 0.06 & 0.37 \\
\hline Garlic & 0.29 & -0.14 & -0.09 \\
\hline Whole grains & 0.28 & -0.18 & -0.08 \\
\hline High-fat dairy products & -0.26 & 0.20 & 0.03 \\
\hline Low-fat dairy products & -0.05 & 0.02 & -0.06 \\
\hline Refined grains & $0 \cdot 16$ & 0.13 & -0.01 \\
\hline Other breakfast cereals & 0.05 & 0.13 & 0.03 \\
\hline Red meats & -0.06 & 0.76 & 0.05 \\
\hline Poultry & $0 \cdot 10$ & 0.71 & 0.14 \\
\hline Meat pies & -0.13 & 0.06 & 0.66 \\
\hline Hamburgers & -0.10 & $0 \cdot 11$ & 0.66 \\
\hline Butter & -0.02 & 0.03 & 0.06 \\
\hline Margarine & -0.06 & 0.06 & 0.02 \\
\hline Eggs & 0.07 & 0.13 & 0.06 \\
\hline Processed meats & -0.08 & 0.51 & 0.38 \\
\hline Fish & 0.09 & 0.32 & -0.004 \\
\hline Potatoes & 0.04 & 0.50 & -0.08 \\
\hline Chips & -0.19 & 0.31 & -0.04 \\
\hline Sweets & -0.02 & -0.05 & 0.44 \\
\hline Condiments & -0.03 & 0.08 & 0.15 \\
\hline Pizza & 0.003 & -0.09 & 0.24 \\
\hline Fruit juice & 0.15 & 0.09 & 0.42 \\
\hline Spirits & 0.11 & 0.24 & 0.28 \\
\hline Beer & 0.04 & 0.03 & 0.46 \\
\hline Wine & -0.08 & 0.08 & 0.06 \\
\hline Variance (\%) & $7 \cdot 6$ & $7 \cdot 6$ & $6 \cdot 3$ \\
\hline
\end{tabular}

(SD 81) and 58 (SD 57) for the Healthy, high protein, high fat and processed foods patterns, respectively.

Partial correlation coefficients between each dietary pattern score and energy-adjusted nutrient intake are shown in Table 3. Healthy pattern score was positively correlated with vitamin $\mathrm{E}$ $\left(\begin{array}{ll}r & 0.67)\end{array}\right)$ vitamin $\mathrm{C}(r \quad 0.45), \mathrm{Mg}(r \quad 0.45), \mathrm{Fe}(r \quad 0.33)$ and carbohydrate $(r 0 \cdot 26)$ but negatively correlated with fat $(r-0 \cdot 32)$ and cholesterol $(r-0 \cdot 22)$. High protein, high fat pattern score was positively correlated with $\mathrm{Zn}(r 0.49)$ cholesterol $(r 0.45)$, protein $(r 0.41)$ and fat $(r 0.29)$ but negatively associated with carbohydrate $(r-0.47)$ and vitamin $\mathrm{E}(r-0 \cdot 23)$. Processed foods pattern was negatively correlated with $\mathrm{Mg}(r-0 \cdot 29), \mathrm{P}(r-0 \cdot 28)$, $\mathrm{Zn}(r-0 \cdot 22)$ and protein $(r-0 \cdot 21)$.

Associations between dietary patterns and musculoskeletal outcomes are shown in Table 4. In univariable analysis healthy pattern score was associated with LMS and FRT. These did not persist after adjustment for our identified confounders, though a borderline significant positively association with LMS remained $(\beta=2 \cdot 8 ; 95 \%$ CI $-0.04,5.7 ; P=0.054)$. LMS was approximately $3.7 \%$ higher for each standard deviation (122 unit) increase in score. Processed foods pattern score was significantly inversely associated with TB BMC $(\beta=-32 \cdot 2 ; 95 \% \mathrm{CI}-59 \cdot 0,-5 \cdot 4)$ and FRT ( $\beta=-0.78 ; 95 \% \mathrm{CI}-1.47,-0.08)$ but no other outcomes. These equate to approximately 1.4 and $1.9 \%$ lower TB BMC
Table 3. Partial Pearson's correlation coefficients between each of three dietary patterns, serum 25-hydroxyvitamin D concentrations and energyadjusted daily nutrient intakes

\begin{tabular}{lccc}
\hline & $\begin{array}{c}\text { 'Healthy' } \\
\text { pattern }\end{array}$ & $\begin{array}{c}\text { 'High protein, } \\
\text { high fat' pattern }\end{array}$ & $\begin{array}{c}\text { 'Processed } \\
\text { foods' pattern }\end{array}$ \\
\hline $\begin{array}{l}\text { Serum 25-hydroxyvitamin D } \\
\text { (nmol/l) }\end{array}$ & 0.12 & -0.11 & -0.06 \\
Energy (MJ) & $0.23^{*}$ & $0.61^{*}$ & $0.41^{*}$ \\
Nutrients & & & \\
Carbohydrate (\% El) & $0.26^{*}$ & $-0.47^{*}$ & 0.13 \\
Protein (\% El) & -0.04 & $0.41^{*}$ & $-0.21^{*}$ \\
Fat (\% El) & $-0.32^{*}$ & $0.29^{*}$ & 0.01 \\
Ca (mg/d) & 0.04 & -0.09 & -0.06 \\
Cholesterol (mg/d) & $-0.22^{*}$ & $0.45^{*}$ & -0.14 \\
Fe (mg/d) & $0.33^{*}$ & -0.09 & -0.12 \\
Mg (mg/d) & $0.45^{*}$ & -0.18 & $-0.29^{*}$ \\
P (mg/d) & 0.12 & 0.11 & $-0.28^{*}$ \\
Na (mg/d) & -0.19 & 0.17 & 0.11 \\
Vitamin C (mg/d) & $0.45^{*}$ & 0.03 & 0.15 \\
Vitamin E (mg/d) & $0.67^{*}$ & $-0.23^{*}$ & -0.12 \\
Zn (mg/d) & -0.10 & $0.49^{*}$ & $-0.22^{*}$ \\
\hline
\end{tabular}

El, energy intake.

* Statistically significant $(P<0.05)$.

and FRT, respectively, for each standard deviation (57 unit) increase in dietary pattern score. There were no associations of high protein, high fat score with any outcome in adjusted models. All associations were not statistically significant when the threshold of significance accounting for multiple testing was applied.

\section{Discussion}

Few studies have examined the association of dietary patterns with bone mass in middle-aged women. To our knowledge, none have examined associations with other musculoskeletal outcomes of muscle strength and balance. The 'Healthy' dietary pattern, more plant-based and characterised by high consumption of vegetables, legumes, fruit, tomatoes, nuts, snacks, garlic, whole grains, was positively associated with $3.7 \%$ higher LMS for each standard deviation increase in dietary pattern score. In contrast, the 'Processed foods' dietary pattern consisting of high intakes of meat pies, hamburgers, beer, sweets, fruit juice, processed meats, snacks, spirits, pizza and low intakes of cruciferous vegetables was significantly and inversely associated with 1.4 and $1.9 \%$ lower TB BMC and FRT for each standard deviation increase in dietary pattern score. These findings suggest that maintaining a healthy diet may be important for TB BMC, muscle strength and balance early in adult life, but do not support the presence of a significant impact of these patterns on BMD in younger women. However, the exploratory nature of the analyses means that confirmation in longitudinal studies and/or trials with prespecified hypotheses is needed.

The association of our Healthy dietary pattern characterised by high consumption of fruit and vegetables with LMS might be clinically important as age-related loss in muscle strength and balance begins to accelerate between $40 \mathrm{~s}$ and $50 \mathrm{~s}^{(14)}$, and estimated annualised rates of loss are 2.2 and $2.5 \%$ for grip strength in women aged 50 and 60 years, respectively ${ }^{(41)}$. However, this should be confirmed by further studies with larger sample size as 
Table 4. Linear regression for associations between three dietary patterns ('Healthy, 'high protein, high fat' and 'Processed foods') and multiple musculoskeletal outcomes ( $\beta$-Coefficients and $95 \%$ confidence intervals)

\begin{tabular}{|c|c|c|c|c|c|}
\hline & \multirow[b]{2}{*}{$n$} & \multicolumn{2}{|c|}{ Unadjusted } & \multicolumn{2}{|c|}{ Adjusted } \\
\hline & & $\beta \ddagger$ & $95 \% \mathrm{Cl}$ & $\beta \ddagger$ & $95 \% \mathrm{Cl}$ \\
\hline \multicolumn{6}{|l|}{ Healthy } \\
\hline TB BMC $(\mathrm{g}) \S$ & 347 & $4 \cdot 2$ & $-30.0,38.5$ & 11.5 & $-14 \cdot 8,37 \cdot 8$ \\
\hline Femoral neck BMD $\left(\mathrm{g} / \mathrm{cm}^{2}\right) \S$ & 347 & -0.002 & $-0.015,0.011$ & 0.002 & $-0.010,0.014$ \\
\hline Lumbar spine BMD $\left(\mathrm{g} / \mathrm{cm}^{2}\right) \S$ & 347 & -0.013 & $-0.029,0.003$ & -0.005 & $-0.021,0.010$ \\
\hline Lower limb muscle strength $(\mathrm{kg}) \|$ & 345 & $3.9^{*}$ & $1 \cdot 2,6 \cdot 6$ & $2.8 \dagger$ & $-0.04,5 \cdot 7$ \\
\hline Timed up and go test (s) $\mathbb{T}$ & 346 & -0.07 & $-0.14,0.01$ & -0.01 & $-0.09,0.06$ \\
\hline Step test (steps)ף & 346 & 0.20 & $-0.07,0.48$ & 0.08 & $-0.21,0.37$ \\
\hline Functional reach test $(\mathrm{cm}) \boldsymbol{\Phi}$ & 346 & $0.73^{\star}$ & $0.06,1.40$ & 0.59 & $-0.10,1.29$ \\
\hline Lateral reach test $(\mathrm{cm}) \boldsymbol{\Upsilon}$ & 344 & 0.13 & $-0.29,0.56$ & 0.14 & $-0.33,0.60$ \\
\hline \multicolumn{6}{|l|}{ High protein, high fat } \\
\hline TB BMC $(\mathrm{g}) \S$ & 347 & $22 \cdot 2$ & $-12 \cdot 0,56 \cdot 4$ & $4 \cdot 3$ & $-27 \cdot 1,35 \cdot 7$ \\
\hline Femoral neck BMD $\left(\mathrm{g} / \mathrm{cm}^{2}\right) \S$ & 347 & $0.014^{*}$ & $0.001,0.027$ & -0.001 & $-0.015,0.013$ \\
\hline Lumbar spine BMD $\left(\mathrm{g} / \mathrm{cm}^{2}\right) \S$ & 347 & 0.013 & $-0.002,0.029$ & -0.00001 & $-0.019,0.019$ \\
\hline Lower limb muscle strength $(\mathrm{kg}) \|$ & 345 & -0.2 & $-2 \cdot 9,2.5$ & -0.8 & $-4 \cdot 2,2 \cdot 5$ \\
\hline Timed up and go test (s) $\boldsymbol{\Pi}$ & 346 & 0.05 & $-0.03,0.12$ & -0.002 & $-0.09,0.08$ \\
\hline Step test (steps)ף & 346 & -0.25 & $-0.52,0.03$ & -0.13 & $-0.47,0.20$ \\
\hline Functional reach test $(\mathrm{cm}) \boldsymbol{\Phi}$ & 346 & -0.39 & $-1.06,0.27$ & -0.47 & $-1.27,0.34$ \\
\hline Lateral reach test $(\mathrm{cm}) \pi$ & 344 & 0.12 & $-0.30,0.53$ & 0.30 & $-0.23,0.83$ \\
\hline \multicolumn{6}{|l|}{ Processed foods } \\
\hline TB BMC $(\mathrm{g}) \S$ & 347 & -0.8 & $-35 \cdot 0,33 \cdot 5$ & $-32 \cdot 2^{*}$ & $-59 \cdot 0,-5 \cdot 4$ \\
\hline Femoral neck BMD $\left(\mathrm{g} / \mathrm{cm}^{2}\right) \S$ & 347 & 0.004 & $-0.009,0.017$ & -0.007 & $-0.019,0.005$ \\
\hline Lumbar spine BMD $\left(\mathrm{g} / \mathrm{cm}^{2}\right) \S$ & 347 & 0.013 & $-0.003,0.029$ & 0.001 & $-0.015,0.016$ \\
\hline Lower limb muscle strength $(\mathrm{kg}) \|$ & 345 & $-1 \cdot 1$ & $-3 \cdot 7,1 \cdot 6$ & $-1 \cdot 8$ & $-4 \cdot 7,1 \cdot 2$ \\
\hline Timed up and go test (s) $\mathbb{T}$ & 346 & -0.001 & $-0.08,0.07$ & -0.02 & $-0.10,0.05$ \\
\hline Step test (steps) & 346 & 0.01 & $-0.26,0.29$ & 0.05 & $-0.24,0.35$ \\
\hline Functional reach test $(\mathrm{cm}) \boldsymbol{\uparrow}$ & 346 & -0.44 & $-1.11,0.23$ & $-0.78^{\star}$ & $-1.47,-0.08$ \\
\hline Lateral reach test $(\mathrm{cm}) \boldsymbol{\Phi}$ & 344 & -0.06 & $-0.48,0.36$ & -0.10 & $-0.56,0.37$ \\
\hline
\end{tabular}

TB BMC, total body bone mineral content; BMD, bone mineral density.

* $P<0.05$.

$+P=0.054$.

$\ddagger \beta$-Coefficients are the change in the outcome for a sD-unit increase in each factor score (i.e. 122,81 and 57 for dietary pattern 1,2 and 3 , respectively). $\S$ Adjusted for weight, height, strenuous physical activity, smoking, total energy intake, Ca and vitamin D supplement and menopausal status.

|| Adjusted for age, weight, height, strenuous physical activity, employment status, hours of watching television, total energy intake and Ca and vitamin D supplement.

I Adjusted for age, weight, height, strenuous physical activity, educational level, hours of watching television, total energy intake and Ca and vitamin D supplement.

the finding was no longer statistically significant after full adjustment of confounders and multiple testing. Previous to our study, an association with such a dietary pattern has only been reported in older adults. A cross-sectional analysis of the Hertfordshire Cohort Study found that a dietary pattern high in fruit and vegetable consumption was positively associated with grip strength in men and women aged $59-73$ years ${ }^{(14)}$. The link between fruit and vegetable intake and muscle strength in older adults has been further confirmed by a recent randomised controlled trial, in eighty-three participants aged 65-85 years comparing habitual $\operatorname{diet}(\leq 2$ portions of fruit and vegetables per $\mathrm{d}$ ) with consumption of $\geq 5$ portions of fruit and vegetable per $\mathrm{d}$ for 16 weeks ${ }^{(42)}$. Participants in the 5 portions/d group had improved grip strength compared with those in the 2 portions/d group that approached significance (mean $=2.04 \quad(\mathrm{SD}$ 5.16) and 0.11 (SD 3.26$) \mathrm{kg}$, respectively, $P=0.06$ ). The benefits of high consumption of fruit and vegetables are biologically plausible as fruit and vegetables contain antioxidants (e.g. vitamins $\mathrm{C}$ and $\mathrm{E})^{(43)}$, which may protect against catabolic effects of oxidative stress on skeletal muscle ${ }^{(44)}$. In our study, a 'Healthy' dietary pattern was strongly correlated with intake of both vitamin $\mathrm{C}$ and $\mathrm{E}$. In older people, low plasma levels of vitamin $\mathrm{E}$ and carotenoids and low dietary consumption of vitamin $\mathrm{C}$ and $\beta$-carotene have been shown to be related to reduced muscle strength ${ }^{(45)}$. These data in older adults, together with our findings in middle-aged women, suggest it is likely that the benefits for maintaining a 'Healthy' dietary pattern on muscle strength are likely to begin earlier rather than later in adult life.

The inverse relationship between a 'processed foods' dietary pattern and FRT was novel and the effect size was of a magnitude that could reach clinical significance, given that annualised rates of deterioration in balance as assessed by Romberg test are 0.3 and $1.6 \%$ in women aged 50 and 60 years, respectively $^{(41)}$. However it is not clear why there were no associations of this dietary pattern with other balance measures. Given that this is an isolated finding from a battery of four balance tests, and the lack of data to support a direct link between the FRT in isolation and clinically important outcomes such as falls or fracture, the clinical relevance of this finding is uncertain. Nonetheless, it is biologically feasible that dietary patterns may influence balance, by effects on skeletal muscle $^{(46,47)}$, visual function ${ }^{(48-50)}$ and reaction time ${ }^{(51)}$. Further studies to confirm links between dietary patterns and balance in middle-aged women are therefore warranted. 
In contrast to our study, a study in premenopausal Japanese women reported associations between a dietary pattern high in fruit and vegetable intakes and forearm $\mathrm{BMD}^{(20)}$. In the Japanese study, the dietary pattern consisted of fish and shellfish, fruit, processed fish, seaweed and soya products in addition to green and white vegetables, mushrooms and fruit. Thus, while this pattern shared fruit and vegetables as major constituents, potential differences include the phyto-oestrogen content from soya (e.g. soya isoflavones) ${ }^{(52,53)}$, and protein and other nutrients from seafood (such as fatty acids) ${ }^{(54)}$ in the Japanese study, both of which may be beneficial for bone health. There were also moderate correlations between the dietary pattern and intakes of Ca $(r 0.51)$ and vitamin D (from fish, shellfish, seaweed and soya) $(r 0.53)$ in the Japanese study but not in ours (see Table 4). In fact, in our study high-fat dairy products loaded negatively on the healthy pattern and low-fat dairy products did not contribute to the pattern score resulting in very low correlation with Ca intake. Both Ca and vitamin D are nutrients important for bone health and overall this, suggests that a dietary pattern high solely in fruit and vegetables may not be optimal for bone health.

Conflicting conclusions have been drawn in the literature on the relationship between a 'Western' dietary pattern and bone health among younger ${ }^{(17,20)}$ and older adults ${ }^{(17-19)}$. Our findings for the 'processed foods' and 'high protein, high fat' pattern (similar to the 'Western' pattern in terms of higher in animal sources of protein) are consistent with those in younger but not older participants of a study demonstrating that a pattern high in soft drinks, potato crisps, French fries, processed meats and desserts was not associated with FN BMD in premenopausal women or younger men but had inverse associations in postmenopausal women and older men (>50 years) ${ }^{(18)}$. Similarly, McNaughton et al. ${ }^{(17)}$ identified a dietary pattern high in energy-dense, nutrient-poor foods (refined cereals, soft drinks, fried potatoes, sausages and processed meat, vegetable oils, beer and take-away foods) that was not associated with BMD at LS or total hip in women aged 18-65 years; however, there was an inverse association between this pattern and TB BMC, which is consistent with our findings for the processed pattern. Interestingly, a study of Japanese women aged 40-55 years showed that a 'Western' dietary pattern (high in meats, processed meats, fats and oils) was inversely but not statistically significantly associated with forearm BMD, but TB BMC was not examined in this study ${ }^{(20)}$. Of note, the negative association of processed foods with bone health might be explained by the finding that protein from other foods, such as low-fat milk, is more beneficial than that from processed foods ${ }^{(55)}$. Potential reasons for this might include the different amino acid composition of proteins from different sources and the potential synergies of proteins with other nutrients, though in at least one study, patterns of dietary intake by protein food group were not associated with bone density or muscle measures whereas total protein intake was positively associated with muscle measures but not BMD ${ }^{(56)}$. Some of the inconsistency in these findings may be due to the patterns not being directly comparable in terms of the food groups contributing to each pattern, as well as differences in populations and study design including age, ethnicity, other lifestyle factors and site at which BMD was measured. Importantly, longitudinal studies are needed to demonstrate the impacts of those dietary patterns on the progression of musculoskeletal health outcomes.
We did not observe associations between the 'high protein, high fat' or 'processed foods' dietary patterns and LMS. Studies specifically examining associations between a 'processed foods' dietary pattern and muscle strength have not been previously reported, but individual nutrients have been associated with muscle function. For example, intervention studies have shown benefits of increased vitamin D status on muscle strength in older adults, though little research has been conducted in younger adults ${ }^{(57)}$. Low protein intake is associated with impaired physical function and the US Health, Aging and Body Composition Study found that both men and women (aged 70-79 years) in the highest quintile of protein intake lost approximately $40 \%$ less lean mass and appendicular lean mass compared with those in the lowest quintile over 3 years ${ }^{(47)}$. However, a prospective study of $2 \cdot 6$ years found no association between nutrient intake and muscle strength of the knee extensors in older adults aged 50-79 years, though a number of nutrients were positive predictors of change in appendicular lean mass $(\mathrm{Mg}(\beta=0.07, P=0.02), \mathrm{P}(\beta=0.07, P=0.047)$ and $\mathrm{Zn}$ $(\beta=0.08, P=0 \cdot 02))^{(46)}$. The authors speculated that diet alone may not offset age-related strength declines and factors such as physical activity are more important for maintenance of muscle function in older adults. Arguably, this could also be the case in our study of middle-aged women.

Our study has limitations. This was an exploratory analysis, and if thresholds for significance that accounted for multiple comparisons were applied, the results were not statistically significant. However, the effect sizes are potentially clinically important, and this arguably warrants more emphasis than $P$ values in exploratory studies ${ }^{(58)}$. Nonetheless, this is a limitation and these results require confirmation in longitudinal studies and/or trials with pre-specified hypotheses. The CCV FFQ does not measure soft drink intake, which may have effects on musculoskeletal health outcomes ${ }^{(59)}$. In addition, FFQ may be subject to recall bias, though the questionnaire we used has been previously validated ${ }^{(30)}$ and has been used for dietary pattern analysis in older populations ${ }^{(38)}$. An objective method such as weighed food records or $24 \mathrm{~h}$ recalls would provide more accurate information on food intake, although logistically more difficult for large-scale studies. The cross-sectional design cannot infer causal associations between dietary patterns, muscle strength and balance, and longitudinal studies are needed. Women who had a higher value of the 'Healthy' dietary pattern might also have a healthier lifestyle as they had a higher level of strenuous physical activity and were more likely to take vitamin D supplements. However, we did a comprehensive selection of confounders and adjusted for physical activity and vitamin D supplement use so the results are independent of these factors. Finally, although the original study ${ }^{(22)}$ had a population-based design, participants were exposed to an osteoporosis behavioural intervention and a dropout rate of $26 \%$ occurred by the end of final follow-up. Some differences in sociodemographic characteristics and smoking behaviour between women retained in the study and those lost to follow-up were observed but the wide spread of education levels at baseline and employment rate at 12 years approximates the overall population figures for these socioeconomic factors and adjustment for potential confounders was made. Therefore our 
findings are likely to apply to healthy middle-aged women from a range of sociodemographic backgrounds.

In conclusion, we identified specific dietary patterns associated with LMS, FRT and TB BMC but not other measures of balance or BMD in middle-aged women. Maintaining a healthy diet may be important for bone acquisition, muscle strength and possibly balance even in early adult life. Our findings should be confirmed in longitudinal studies of larger sample size with prespecified hypotheses. Our findings may provide evidence for developing potential dietary strategies, such as improving intake of fruit, vegetables and whole grains, for improving muscle and balance health in middle-aged women.

\section{Acknowledgements}

The authors thank all the volunteers and participants involved in the present study.

This study was funded by the National Health and Medical Research Council (NHMRC) (APP1003437) and Royal Australian College of General Practitioners (RACGP)/Osteoporosis Australia Bone Health Research Grant. T. W. was supported by a NHMRC/ Primary Health Care Research, Evaluation and Development (PHCRED) Career Development Fellowship (grant no. APP102859) and G. J. is supported by a NHMRC Practitioner Fellowship. L. L. is supported by an Arthritis Foundation Australia - Australian Rheumatology Association Heald Fellowship, funded by the Australian Rheumatology Association and Vincent Fairfax Family Foundation; and a NMHRC Early Career Fellowship (Australian Clinical Research Fellowship) (grant no. APP1070586). They did not have any role in the study concept, design, data analysis, writing of the manuscript or submission of the manuscript for publication. The researchers are totally independent of the funders.

T. W., F. W., G. J., and B. O. were involved in study design. T. W. and G. J. were responsible for data collection and management. F. W. performed data analysis, in consultation with K. W., T. W., L. L. and G. J. F. W. and T. W. drafted the manuscript together. All authors revised manuscript content and approved the final manuscript and had access to the data. T. W. is the guarantor of the study and accepts full responsibility for the finished article, had access to any data and controlled the decision to publish.

All authors have completed the ICMJE uniform disclosure form at www.icmje.org/coi_disclosure.pdf and declare: no support from any organisation for the submitted work; no financial relationships with any organisations that might have an interest in the submitted work in the previous 3 years; no other relationships or activities that could appear to have influenced the submitted work.

The lead author affirms that the manuscript is an honest, accurate and transparent account of the study being reported; that no important aspects of the study have been omitted; and that any discrepancies from the study as planned (and, if relevant, registered) have been explained.

\section{Supplementary material}

For supplementary material/s referred to in this article, please visit https://doi.org/10.1017/S0007114517002483

\section{References}

1. Gold DT (1996) The clinical impact of vertebral fractures: quality of life in women with osteoporosis. Bone 18, 185S-189S

2. Ioannidis G, Papaioannou A, Hopman WM, et al. (2009) Relation between fractures and mortality: results from the Canadian Multicentre Osteoporosis Study. CMAJ 181, 265-271.

3. Grisso JA, Kelsey JL, Strom BL, et al. (1991) Risk factors for falls as a cause of hip fracture in women. The Northeast Hip Fracture Study Group. N Engl J Med 324, 1326-1331.

4. Rubenstein LZ (2006) Falls in older people: epidemiology, risk factors and strategies for prevention. Age Ageing 35, Suppl. 2, ii37-ii41.

5. Tinetti ME, Speechley M \& Ginter SF (1988) Risk factors for falls among elderly persons living in the community. $N$ Engl J Med 319, 1701-1707.

6. Fukagawa NK, Wolfson L, Judge J, et al. (1995) Strength is a major factor in balance, gait, and the occurrence of falls. J Gerontol A Biol Sci Med Sci 50A, 64-67.

7. Puthoff ML \& Nielsen DH (2007) Relationships among impairments in lower-extremity strength and power, functional limitations, and disability in older adults. Phys Ther $\mathbf{8 7}$, 1334-1347.

8. Sayer AA, Syddall HE, Martin HJ, et al. (2006) Falls, sarcopenia, and growth in early life: findings from the Hertfordshire cohort study. Am J Epidemiol 164, 665-671.

9. Moreland JD, Richardson JA, Goldsmith $\mathrm{CH}$, et al. (2004) Muscle weakness and falls in older adults: a systematic review and meta-analysis. J Am Geriatr Soc 52, 1121-1129.

10. El Haber N, Erbas B, Hill KD, et al. (2008) Relationship between age and measures of balance, strength and gait: linear and non-linear analyses. Clin Sci (Lond) 114, 719-727.

11. Choy NL, Brauer S \& Nitz J (2003) Changes in postural stability in women aged 20 to 80 years. J Gerontol A Biol Sci Med Sci 58, 525-530.

12. Palacios C (2006) The role of nutrients in bone health, from A to Z. Crit Rev Food Sci Nutr 46, 621-628.

13. Robinson S, Cooper C \& Aihie Sayer A (2012) Nutrition and sarcopenia: a review of the evidence and implications for preventive strategies. J Aging Res 2012, 510801.

14. Robinson SM, Jameson KA, Batelaan SF, et al. (2008) Diet and its relationship with grip strength in community-dwelling older men and women: the Hertfordshire cohort study. J Am Geriatr Soc 56, 84-90.

15. Reinders I, Murphy RA, Song X, et al. (2015) Polyunsaturated fatty acids in relation to incident mobility disability and decline in gait speed; the Age, Gene/Environment SusceptibilityReykjavik Study. Eur J Clin Nutr 69, 489-493.

16. Kontogianni M \& Yiannakouris N (2009) Diet and bone health the perspective of dietary pattern analysis. Eur Musculoskelet Rev 4, 73-74.

17. McNaughton SA, Wattanapenpaiboon $\mathrm{N}$, Wark JD, et al. (2011) An energy-dense, nutrient-poor dietary pattern is inversely associated with bone health in women. J Nutr $\mathbf{1 4 1}$, $1516-1523$

18. Langsetmo L, Poliquin S, Hanley DA, et al. (2010) Dietary patterns in Canadian men and women ages 25 and older: relationship to demographics, body mass index, and bone mineral density. BMC Musculoskelet Disord 11, 20.

19. Hardcastle AC, Aucott L, Fraser WD, et al. (2011) Dietary patterns, bone resorption and bone mineral density in early postmenopausal Scottish women. Eur J Clin Nutr 65, 378-385.

20. Okubo H, Sasaki S, Horiguchi H, et al. (2006) Dietary patterns associated with bone mineral density in premenopausal Japanese farmwomen. Am J Clin Nutr 83, 1185-1192. 
21. Whittle CR, Woodside JV, Cardwell CR, et al. (2012) Dietary patterns and bone mineral status in young adults: the Northern Ireland Young Hearts Project. Br J Nutr 108, 1494-1504.

22. Winzenberg TM, Oldenburg B, Frendin S, et al. (2005) Effects of bone density feedback and group education on osteoporosis knowledge and osteoporosis self-efficacy in premenopausal women: a randomized controlled trial. J Clin Densitom 8, 95-103.

23. Isles RC, Choy NL, Steer M, et al. (2004) Normal values of balance tests in women aged 20-80. J Am Geriatr Soc 52, 1367-1372.

24. Duncan PW, Weiner DK, Chandler J, et al. (1990) Functional reach: a new clinical measure of balance. J Gerontol $\mathbf{4 5}$, M192-M197.

25. Brauer S, Burns Y \& Galley P (1999) Lateral reach: a clinical measure of medio-lateral postural stability. Physiother Res Int 4, 81-88.

26. Podsiadlo D \& Richardson S (1991) The timed 'Up \& Go': a test of basic functional mobility for frail elderly persons. $J \mathrm{Am}$ Geriatr Soc 39, 142-148.

27. Hill KD, Bernhardt J, McGann AM, et al. (1996) A new test of dynamic standing balance for stroke patients: reliability, validity and comparison with healthy elderly. Physiother Can 48, 257-262.

28. Dore D, Quinn S, Ding C, et al. (2010) Natural history and clinical significance of MRI-detected bone marrow lesions at the knee: a prospective study in community dwelling older adults. Arthritis Res Ther 12, R223.

29. Shrout PE \& Fleiss JL (1979) Intraclass correlations: uses in assessing rater reliability. Psychol Bull 86, 420-428.

30. Keogh JB, Lange K \& Syrette J (2010) Comparative analysis of two FFQ. Public Health Nutr 13, 1553-1558.

31. Hodge A, Patterson AJ, Brown WJ, et al. (2000) The Anti Cancer Council of Victoria FFQ: relative validity of nutrient intakes compared with weighed food records in young to middle-aged women in a study of iron supplementation. Aust N Z J Public Health 24, 576-583.

32. English R \& Lewis J (1991) Nutritional Values of Australian Foods. Canberra: AGPS.

33. Aaron DJ, Kriska AM, Dearwater SR, et al. (1995) Reproducibility and validity of an epidemiologic questionnaire to assess past year physical activity in adolescents. Am J Epidemiol 142, 191-201.

34. Jones G \& Scott FS (1999) A cross-sectional study of smoking and bone mineral density in premenopausal parous women: effect of body mass index, breastfeeding, and sports participation. J Bone Miner Res 14, 1628-1633.

35. Mezquita-Raya P, Munoz-Torres M, Luna JD, et al. (2001) Relation between vitamin D insufficiency, bone density, and bone metabolism in healthy postmenopausal women. J Bone Miner Res 16, 1408-1415.

36. Mowe M, Haug E \& Bohmer T (1999) Low serum calcidiol concentration in older adults with reduced muscular function. J Am Geriatr Soc 47, 220-226.

37. Gerdhem P, Ringsberg KA, Obrant KJ, et al. (2005) Association between 25-hydroxy vitamin D levels, physical activity, muscle strength and fractures in the prospective population-based OPRA Study of Elderly Women. Osteoporos Int 16, 1425-1431.

38. Gardener SL, Rainey-Smith SR, Barnes MB, et al. (2015) Dietary patterns and cognitive decline in an Australian study of ageing. Mol Psychiatry 20, 860-866.

39. Slattery ML \& Boucher KM (1998) The senior authors' response: factor analysis as a tool for evaluating eating patterns. Am J Epidemiol 148, 20-21.

40. Wu F, Laslett LL, Wills K, et al. (2014) Effects of individualized bone density feedback and educational interventions on osteoporosis knowledge and self-efficacy: a 12-yr prospective study. J Clin Densitom 17, 466-472.

41. Daly RM, Rosengren BE, Alwis G, et al. (2013) Gender specific age-related changes in bone density, muscle strength and functional performance in the elderly: a-10 year prospective population-based study. BMC Geriatr 13, 71.

42. Neville CE, Young IS, Gilchrist SE, et al. (2013) Effect of increased fruit and vegetable consumption on physical function and muscle strength in older adults. Age (Dordr) 35, 2409-2422.

43. Wu X, Beecher GR, Holden JM, et al. (2004) Lipophilic and hydrophilic antioxidant capacities of common foods in the United States. J Agric Food Chem 52, 4026-4037.

44. Doria E, Buonocore D, Focarelli A, et al. (2012) Relationship between human aging muscle and oxidative system pathway. Oxid Med Cell Longev 2012, 830257.

45. Cesari M, Pahor M, Bartali B, et al. (2004) Antioxidants and physical performance in elderly persons: the Invecchiare in Chianti (InCHIANTI) study. Am J Clin Nutr 79, 289-294.

46. Scott D, Blizzard L, Fell J, et al. (2010) Associations between dietary nutrient intake and muscle mass and strength in community-dwelling older adults: the Tasmanian Older Adult Cohort Study. J Am Geriatr Soc 58, 2129-2134.

47. Houston DK, Nicklas BJ, Ding J, et al. (2008) Dietary protein intake is associated with lean mass change in older, community-dwelling adults: the Health, Aging, and Body Composition (Health ABC) Study. Am J Clin Nutr 87, 150-155.

48. Yao Y, Qiu QH, Wu XW, et al. (2013) Lutein supplementation improves visual performance in Chinese drivers: 1-year randomized, double-blind, placebo-controlled study. Nutrition 29, 958-964.

49. Ma L, Yan SF, Huang YM, et al. (2012) Effect of lutein and zeaxanthin on macular pigment and visual function in patients with early age-related macular degeneration. Ophthalmology 119, 2290-2297.

50. Renzi LM, Bovier ER \& Hammond BR Jr. (2013) A role for the macular carotenoids in visual motor response. Nutr Neurosci 16, 262-268.

51. Bovier ER, Renzi LM \& Hammond BR (2014) A double-blind, placebo-controlled study on the effects of lutein and zeaxanthin on neural processing speed and efficiency. PLOS ONE 9, $\mathrm{e} 108178$.

52. Patisaul HB \& Jefferson W (2010) The pros and cons of phytoestrogens. Front Neuroendocrinol 31, 400-419.

53. Greendale GA, FitzGerald G, Huang MH, et al. (2002) Dietary soy isoflavones and bone mineral density: results from the study of women's health across the nation. Am J Epidemiol 155, 746-754.

54. Zalloua PA, Hsu YH, Terwedow H, et al. (2007) Impact of seafood and fruit consumption on bone mineral density. Maturitas 56, 1-11.

55. Mangano KM, Sahni S, Kiel DP, et al. (2015) Bone mineral density and protein-derived food clusters from the Framingham Offspring Study. J Acad Nutr Diet 115, 1605-1613, e1601.

56. Mangano KM, Sahni S, Kiel DP, et al. (2017) Dietary protein is associated with musculoskeletal health independently of dietary pattern: the Framingham Third Generation Study. Am J Clin Nutr 105, 714-722.

57. McCarthy EK \& Kiely M (2015) Vitamin D and muscle strength throughout the life course: a review of epidemiological and intervention studies. J Hum Nutr Diet 28, 636-645.

58. Bender R \& Lange S (2001) Adjusting for multiple testing when and how? J Clin Epidemiol 54, 343-349.

59. Tucker KL, Morita K, Qiao N, et al. (2006) Colas, but not other carbonated beverages, are associated with low bone mineral density in older women: The Framingham Osteoporosis Study. Am J Clin Nutr 84, 936-942. 